\title{
OS EFEITOS DA EQUOTERAPIA NO EQUILÍBRIO DE PRATICANTES COM SÍNDROME DE DOWN
}

DOI: 10.22289/2446-922X.V4N1A7

\author{
Tatiane dos Reis Fernandes ${ }^{1}$ \\ Lacyelle Lúcia de Souza \\ Mariane Fernandes Ribeiro
}

\section{RESUMO}

A síndrome de Down apresenta algumas características particulares, e dentre elas, o déficit de equilíbrio é uma das consequências. A equoterapia é um método terapêutico que vem sendo utilizada por trazer benefícios a estes pacientes, incluindo a melhora do equilíbrio. $O$ atual artigo foi realizado para averiguar os efeitos da equoterapia e sua melhora do quadro de equilíbrio em indivíduos com síndrome de Down. Foi realizada a busca pela base de dados: PubMed / LiLacs, SciELO, PEDro, utilizando as palavras-chaves: equoterapia, equilíbrio e síndrome de Down, bem como os seus correspondentes em inglês: hippotherapy, equilibrium, Down syndrome, utilizadas isoladamente ou em conjunto. Foram incluídos artigos entre os anos de 2006 a 2017, que relatam a melhora do equilíbrio em Síndrome de Down. Foram encontrados 492 artigos, e dentre eles, 5 se enquadravam nos parâmetros necessários para inclusão nesta revisão, sendo excluídos os demais. A maioria dos autores defenderam que a equoterapia vem se desenvolvendo tanto para ganhos de equilíbrio quanto outros ganhos motores, obtendo resultados significantes em adaptação do praticante. Verificou-se que a montaria com o mante e pé fora do estribo leva à uma maior atividade muscular, e que atividades lúdicas podem complementar o atendimento. Como resultados finais verificou-se que a prática de equoterapia trás grandes benefícios para paciente com síndrome de Down em relação ao equilíbrio estático e dinâmico, podendo também ser utilizada em outras patologias.

Palavras-chave: Terapia Assistida por cavalos; Equilíbrio; Síndrome de Down.

\begin{abstract}
The deficit of balance is one of the consequences of the alteration of Down syndrome among others. Hippotherapy is a technique that has been widely used just because it is not a conventional technique. The current article was carried out to investigate the effects of equine therapy and its improvement in the balance improvement in individuals with Down syndrome, showing some techniques used and highlighting which is the most effective. We searched the database: PubMed / LiLacs, SciELO, PEDro, used the keywords: hippotherapy, equilibrium,
\end{abstract}

\footnotetext{
${ }^{1}$ Endereço eletrônico de contato: tatyannereiz123@hotmail.com

Recebido em 01/11/2017. Aprovado pelo conselho editorial para publicação em 14/11/2017.
}

Rev. Psicol Saúde e Debate. Fev., 2018:4(1):119-129. 
down syndrome used alone or in combination. Articles were included between the years 2006 to 2017 where they report the improvement of the balance in Down Syndrome. A total of 492 articles were found, of which 5 were included in the parameters necessary for inclusion in this review, and the others were excluded. The majority of the authors argued that hippotherapy has been developing for both balance gains and other motor gains, obtaining significant results in the adaptation of the practitioner. It was verified that the mount with the mante and foot outside the stirrup leads to a greater muscular activity, and that ludic activities can complement the attendance. As final results it was verified that the practice of equoterapia brings great benefits to patients with Down syndrome in relation to the static and dynamic balance, and it can also be used in other pathologies.

Keywords: Hippotherapy; Equilibrium; Down Syndrome.

\section{INTRODUÇÃO}

A Síndrome de Down (SD) foi relatada em meados de 1866 pelo médico Jonh Langdon Down, porém apenas em 1959, Joreme Lejeune apontou as explicações genéticas (Meneghetti, Porto, Iwabe \& Poletti, 2009). Associada ao atraso intelectual, apresenta-se pela modificação genética, a trissomia do cromossomo 21 . O ser humano possui 46 cromossomos, dos quais são agrupados em 23 pares, no entanto, a pessoa com SD apresenta um cromossomo a mais, portando 47 cromossomos (Barreto, Gomes, Silva \& Gomes, 2007).

Quanto ao diagnóstico, durante o período de gestação, é possível constatar se o feto apresenta a síndrome ou não. Como fator de predisposição são descritos: a gravidez em mulheres acima de 35 anos, que já apresenta outro filho com fator positivo (Barreto et al., 2007). As pessoas com a SD apresentam algumas características distintas de pessoas sem esse comprometimento, como alterações cognitivas, físicas e motoras. No campo de atividades motoras, realizam-nas de forma mais lenta, possuem dificuldades em realizar atividades de precisão, e também aquelas simples do cotidiano (Gimenez, Stefanoni \& Farias, 2007).

Dentre algumas das alterações apresentadas estão a hipoplásica cerebral, causadora da hipotonia, frouxidão ligamentar, alterações biomecânicas que resultam em carência de controle postural, discrepância na densidade óssea, hipoplásica de cartilagem, o que influência na manutenção ou falta de equilíbrio e déficit de força muscular (Espíndula, Ribeiro, Souza, Ferreira \& Teixeira, 2015). Apresentam também problemas auditivos e cardíacos, e uma tendência a desenvolverem obesidade (Borssatti, Anjos \& Ribas, 2013).

Em meio às várias modalidades terapêuticas abordadas em crianças com SD, a equoterapia vem se destacando notavelmente devido à sua contribuição na melhora destes pacientes (Copetti, Mote, Graup, Menezes \& Venturini, 2007) 
A equoterapia já se manifestava desde a antiga Grécia, já descrita por Hipócrates (Granados \& Agís, 2011). Ela caracteriza-se por ser uma técnica terapêutica que se dispõe do cavalo como realizador de ganhos. Nela o equino produz em sua andadura ao passo, um movimento tridimensional, provocando no corpo do indivíduo uma grande quantidade de estímulos, que irão intervir positivamente em habilidades motoras, desenvolvimento global e melhora vida social. Além da SD, outras patologias utilizam a equoterapia como método terapêutico (Cardozo \& Vespasiano, 2012).

O equino apresenta três tipos de andaduras: passo, trote, galope. De forma que é durante a alternação dos passos, que ele produz os movimentos tridimensionais e oscilações aos quais o praticante precisa se adaptar. Geralmente mesmo parado ele não se apresenta totalmente estático, há mudanças de localização e trocas de membros, e movimentos com a cabeça para direita e esquerda. Trote e galope são passadas saltadas, é exigido ao praticante um estímulo maior, apresenta movimentos mais ágeis, pois o cavalo não toca as patas ao chão. O passo é a andadura, utilizadas em programas de reabilitação, são passos rolados ou marchados, onde se encontra alguns dos membros em contato com o solo, é uma marcha ritmada, em quatro tempos de candência. A partir do movimento realizado de um lado do animal, o mesmo é repetido pelo outro lado, tornando assim movimentos simétricos, mais lentos, assim sobressaindo maiores resultados aos praticantes (Liporoni \& Oliveira, 2005).

O cavalo é utilizado como promotor de ganhos tanto físicos, quanto educacionais e psicológicos, e a terapia vem sendo aperfeiçoada por meio de estudos e pesquisas, embora ainda necessite de mais aprendizado.(8) Nesse contexto, destaca-se como um método terapêutico com abordagem multidisciplinar nas áreas de educação, equitação e saúde, contribuindo para a melhor independência das pessoas com necessidades especiais, junto a benefícios como: melhora da coordenação motora, e equilíbrio estático e dinâmico (Liporoni \& Oliveira, 2005).

A prática é realizada ao ar livre, o animal deverá ser dócil para que o praticante estabeleça um laço afetivo com o equino, assim despertando a questão afetiva e emocional, e tornando o atendimento mais lúdico, mais prazeroso, proporcionando assim ao praticante um contato maior com a natureza (Cardozo \& Vespasiano, 2012).

Estudos apontam que o efeito tridimensional imposto pelo andar do cavalo, exerce sobre a cintura pélvica um deslocamento de $5 \mathrm{~cm}$ em planos verticais, bem como uma rotação na horizontal de 8 graus de um lado para outro. Indica-se que 30 minutos da prática de equoterapia fornece entre 1800 a 2250 ajustamentos tônicos (Silva \& Aguiar, 2008).

O tema foi abordado para que possa proporcionar maior conhecimento deste recurso, como é realizado, quais as atividades podem ser abordadas, os movimentos e o efeito 
fisiológico promovidos no praticante com Síndrome de Down e sobretudo para a melhora de seu equilíbrio estático e dinâmico. A SD encontra-se muito presente atualmente, e esse trabalho foi realizado para que se torne um guia para reabilitação complementar, para esclarecer dúvidas a respeito da equoterapia por ser um método terapêutico que demonstra diversos resultados positivos. Diante do que foi ressaltado, essa técnica vem surpreendendo, tanto pela melhora do praticante em seu aspecto motor, quanto nos aspectos psicológicos e comportamentais diante da reabilitação proposta pelo fisioterapeuta em conjunto a uma equipe multidisciplinar.

Sendo assim, essa pesquisa tem como objetivo realizar uma revisão da literatura e identificar os efeitos da equoterapia sobre o equilíbrio em praticantes com Síndrome de Down. Procurou-se asseverar os padrões de melhoras do equilíbrio e relacionar com os protocolos de tratamento equoterapêutico.

\section{METODOLOGIA}

Esse trabalho se caracteriza como uma Revisão da Literatura que buscou qualificar e agrupar amostras científicas relativas as resultâncias da equoterapia diante da alteração de equilíbrio na SD. A busca foi realizada nas bases de dados: PubMed, SciELO, Lilacs, PEDro, de artigos publicados entre os anos de 2007 a 2017. As palavras-chave empregadas foram: equoterapia, equilíbrio e Síndrome de Down, separadamente e combinadas entre si, seus correspondentes em inglês são: hippotherapy, equilibrium, Down Syndrom.

Como critérios de inclusão, foram analisados os artigos que tratassem de pesquisa de campo, avaliassem o equilíbrio estático ou dinâmico em indivíduos com SD de ambos os sexos, submetidos à equoterapia, bem como aqueles que comparavam a equoterapia com outras técnicas na melhora do equilíbrio, e que tivessem acesso disponível ao texto completo nas bases de dados. O processo de inclusão dos estudos se deu por meio da análise do título e resumo dos artigos para informações contidas nas buscas eletrônicas com potencial. Foram excluídos aqueles que utilizaram a equoterapia como forma de tratamento em outras patologias, os que não deixavam claro o protocolo de tratamento equoterapêutico e os que não avaliavam o equilíbrio na SD.

\section{RESULTADOS E DISCUSSÃO}


Foram encontrados no total de 492 artigos. Dentre eles, apenas 5 se enquadravam nos parâmetros necessários para inclusão nesta revisão, sendo excluídos os demais. Os artigos analisados nesta revisão estão expostos no quadro abaixo.

\begin{tabular}{|c|c|c|c|c|}
\hline Autor & Objetivo & Metodologia & Tratamento & Resultados \\
\hline $\begin{array}{l}\text { Copetti et al., } \\
2007\end{array}$ & $\begin{array}{lr}\text { Evolução } & \text { de } \\
\text { repostas } & \text { de } \\
\text { equilíbrio } & \text { e } \\
\text { postural através } \\
\text { da prática de } \\
\text { equoterapia }\end{array}$ & $\begin{array}{l}\text { - } 3 \text { crianças do sexo } \\
\text { masculino com SD } \\
\text { - idade média de } 7,3 \\
\text { anos }\end{array}$ & $\begin{array}{l}\text { - } 13 \text { Sessões, } \\
\text { - } 35 \text { minutos, } \\
\text { - intervalo de } 7 \text { dias } \\
\text { cada sessão }\end{array}$ & \begin{tabular}{lr}
\multicolumn{3}{l}{ Benefícios positivos } \\
associados \\
prática \\
atribuídos \\
estímulos \\
sensoriais. \\
análise de \\
angulares & a \\
tornozelo do \\
joelho após de \\
prática: houve uma \\
melhora \\
significativa.
\end{tabular} \\
\hline $\begin{array}{ll}\text { Lima } & e \\
\text { Miyagawa } & \\
2007 & \end{array}$ & $\begin{array}{lr}\text { Observar } & \text { a } \\
\text { influência } & \text { da } \\
\text { equoterapia } & \text { no } \\
\text { tratamento } & \text { das } \\
\text { alterações } & \text { de } \\
\text { equilíbrio } & \text { em } \\
\text { portadores } & \text { da } \\
\text { síndrome } & \text { de } \\
\text { Down } & \end{array}$ & $\begin{array}{lr}\text { - } 16 \text { indivíduos } \\
\text { - Grupo A: pessoas } \\
\text { com síndrome de } \\
\text { Down } \\
\text { utilizavam que } \\
\text { fisioterapia } \\
-\quad \text { Grupo } \\
\text { praticavam } \\
\text { equoterapia ha } \\
\text { mais de } 6 \text { meses } \\
\text { - } 8 \text { a } 30 \text { anos } \\
\end{array}$ & $\begin{array}{l}\text { - Aplicado } 0 \\
\text { protocolo criado por } \\
\text { O'Sullivan com } 10 \\
\text { testes de equilíbrio } \\
\text { - } 15 \text { segundos para } \\
\text { realizar cada teste } \\
\text { - Utilizada uma } \\
\text { escala } \\
\text { pontuação de } 0 \text { a } 4\end{array}$ & $\begin{array}{l}\text { Conclui-se que a } \\
\text { equoterapia e seus } \\
\text { estímulos motores } \\
\text { ajudam na melhora } \\
\text { de equilíbrio. }\end{array}$ \\
\hline $\begin{array}{l}\text { Torquato et } \\
\text { al., } \\
2013\end{array}$ & $\begin{array}{l}\text { Verificar a } \\
\text { aquisição de } \\
\text { marcos motores e } \\
\text { equilíbrio em } \\
\text { crianças com SD } \\
\text { que utilizavam a } \\
\text { equoterapia ou } \\
\text { fisioterapia } \\
\text { convencional }\end{array}$ & $\begin{array}{l}\text { - } 33 \text { indivíduos } \\
\text { - Grupo } \\
\text { equoterapia: } 19 \\
\text { crianças, com } \\
\text { média de idade de } \\
7,73( \pm 2,25) \\
\text { - Grupo de } \\
\text { fisioterapia } \\
\text { convencional, } \\
\text { constituído de } \\
14 \text { crianças, com } \\
\text { média de idade de } \\
7,71( \pm 1,43) \text {. } \\
\text { - tempo de } \\
\text { tratamento } \\
\text { fisioterapia: } \\
27( \pm 10,80) \\
\text { meses, grupo } \\
\text { equoterapia } \\
\text { apresentou } 18,10 \pm \\
(11,13) \text { meses. }\end{array}$ & $\begin{array}{l}\text { - Realizaram } 1 \\
\text { avaliação de } \\
\text { equilíbrio estático e } \\
\text { dinâmico } \\
\text {-aplicaram } \\
\text { questionário } \\
\text { biopsicossocial de } \\
\text { desenvolvimento } \\
\text { motor } \\
\text { - exercícios de de } \\
\text { colocar e tirar pés } \\
\text { do estribo } \\
\text { - ficar de olhos } \\
\text { abertos e fechados. }\end{array}$ & $\begin{array}{l}\text {-Verificaram que } \\
\text { quanto mais } \\
\text { precoce for iniciado } \\
\text { o trabalho melhores } \\
\text { resultados } \\
\text { - Fisioterapia houve } \\
\text { maiores ganhos } \\
\text { tanto em equilíbrio } \\
\text { quanto motores } \\
\text { - Equoterapia } \\
\text { houve reações } \\
\text { adaptativas } \\
\text { melhores. }\end{array}$ \\
\hline $\begin{array}{l}\text { Espindula et } \\
\text { al., } 2014\end{array}$ & $\begin{array}{l}\text { - Qual o melhor } \\
\text { material de } \\
\text { montaria e }\end{array}$ & $\begin{array}{l}\text { - } 5 \text { indivíduos } \\
\text { - sexo masculino }\end{array}$ & \begin{tabular}{|l|}
-4 sessões \\
-30 minutos \\
-1 vez na semana
\end{tabular} & $\begin{array}{l}\text {-A utilização da } \\
\text { manta associado } \\
\text { com os pés fora do }\end{array}$ \\
\hline
\end{tabular}

Rev. Psicol Saúde e Debate. Fev., 2018:4(1):119-129. 


\begin{tabular}{|c|c|c|c|c|}
\hline & $\begin{array}{l}\text { posicionamento } \\
\text { dos pés, para } \\
\text { recrutamento de } \\
\text { musculatura de } \\
\text { tronco em } \\
\text { crianças com SD. }\end{array}$ & $\begin{array}{l}\text { - Idade média de } \\
11,2 \text { anos. }\end{array}$ & $\begin{array}{l}-\quad \text { utilizaram a } \\
\text { eletromiografia } \\
-\quad \text { diferentes } \\
\text { materiais de sela e } \\
\text { posicionamento dos } \\
\text { pés }\end{array}$ & $\begin{array}{l}\text { estribo ocasionou } \\
\text { maior recrutamento } \\
\text { dos músculos } \\
\text { estudados, sendo o } \\
\text { músculo da região } \\
\text { cervical o mais } \\
\text { ativado. }\end{array}$ \\
\hline $\begin{array}{l}\text { Schelbauer e } \\
\text { Pereira. } \\
2012\end{array}$ & $\begin{array}{l}\text { Mostrar os efeitos } \\
\text { da equoterapia } \\
\text { diante } \quad \text { dos } \\
\text { pacientes com } \\
\text { SD, juntamente } \\
\text { com a } \\
\text { psicomotricidade }\end{array}$ & $\begin{array}{l}\text { - } 5 \text { indivíduos } \\
-2 \text { a } 11 \text { anos } \\
\text { - ambos sexos }\end{array}$ & 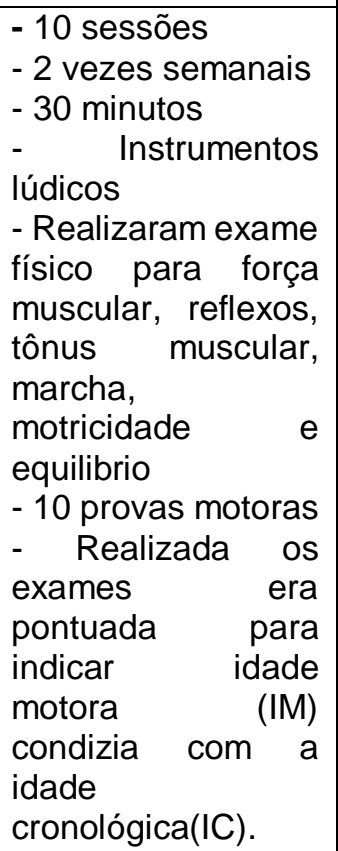 & $\begin{array}{l}\text { Ao final do estudo } \\
\text { mostrou benefícios } \\
\text { significativos na } \\
\text { melhora } \\
\text { equilíbrio, } \\
\text { motricidade, força } \\
\text { muscular, nas fases } \\
\text { da marcha, no } \\
\text { tônus. } \\
\text { - A equoterapia } \\
\text { apresenta um papel } \\
\text { importante no } \\
\text { desenvolvimento } \\
\text { biopsicossocial. }\end{array}$ \\
\hline
\end{tabular}

Quadro 1. Análise descritiva dos resultados

Observou-se que a maioria dos estudos apresentados descreve uma melhora significativa do equilíbrio em praticantes com SD submetidos à equoterapia como modalidade terapêutica e apontam sua eficácia, podendo ser utilizado como método complementar na reabilitação ou ganho e aprimoramento de habilidades motoras nestes indivíduos. Em um estudo realizado por Copetti, Mote, Graup, Menezes e Venturini (2007), avaliou-se o comportamento angular do tornozelo e joelho em indivíduos com SD submetidos à equoterapia, bem como as respostas de equilíbrio, melhora postural e controle de tronco, por meio uma filmagem bidimensional, antes e após a prática. Participaram deste estudo três crianças com SD, nas quais realizaram-se 13 atendimentos de equoterapia, mediante as andaduras de passo e trote em asfalto, gramado e areia, e com equipamentos de montaria a manta, solicitando ao praticante que o pé ficasse no estribo constantemente. Verificaram que houve uma melhora significativa no comportamento angular do tornozelo devido ao aumento de dorsiflexão, pela prática do apoio simples do pé, que é visto como um período instável em 
que o corpo do praticante está em maior desequilíbrio, o centro de gravidade é acelerado para frente e o centro de pressão está embaixo do pé. Melhoras no comportamento angular do joelho também foram apresentadas, porém não houve diferenças estatísticas. Tais modificações no joelho e tornozelo proporcionaram melhora no andar, ajudando também na evolução às reações de equilíbrio e postural.

Durante a montaria, os praticantes tendem a se ajustar a movimentação do cavalo, conseguindo assim equilibrar-se e efetuar movimentos concomitantemente aos do animal, assim fortalecendo a musculatura hipotônica. O ajustamento tônico está entre os principais efeitos no corpo do praticante sobre o dorso do equino, já que o mesmo nunca se encontra estático, sempre apresenta oscilações de movimento. Diante disso todo movimento que o cavalo proporciona ao praticante faz com que haja um ajuste do tónus muscular, devido aos estímulos proprioceptivos, do qual o equino possibilita a propagação destes ao sistema neurológico.

Alguns estudos foram realizados comparando os efeitos da equoterapia e da fisioterapia convencional nas variáveis de equilíbrio de pessoas com SD, e encontraram divergências de resultados. Como em Lima e Miyagawa (2007), os quais realizaram um estudo de análise do grau de equilíbrio de indivíduos com SD diante das técnicas de equoterapia e fisioterapia convencional, ressaltando qual seria a mais eficaz. Foram aplicadas fichas de avaliação contendo 10 diferentes testes de equilíbrio, das quais os participantes teriam 15 segundos para realizarem, assim sendo aplicada uma escala de pontuação de 0 , quando fossem incapazes de realizar a 4, quando realizado normalmente sem esforço. Ao final do estudo comparativo verificou-se uma visível diferença de melhora do equilíbrio em praticantes que utilizavam a equoterapia como instrumento cinesioterapêutico.

Por outro lado, Torquato, Lança, Pereira, Carvalho e Silva (2013), encontrou resultados diferentes em seu estudo, que se deu no período de um mês, sendo utilizado um grupo para fisioterapia convencional e outro para equoterapia. Foi avaliado a aquisição de marcos motores em crianças com SD com características de hipotonia e alteração da acuidade visual. Foi realizada uma única avaliação de equilíbrio estático e dinâmico, aplicaram juntamente um questionário biopsicossocial de escala de desenvolvimento motor (idade em que adquiriu controle cervical, sedestação, rolamento, quatro apoios, postura de semiajoelhado e ajoelhado, engatinhar, ortotatismo, marcha sozinha) entre outras, e empregaram a escala de Daniels para força. Utilizaram atividades para testar equilíbrio estático, motricidade fina e esquema corporal. O teste era realizado de acordo com a idade cronológica. Segundo os autores, somente pelo fato de andar a cavalo, o indivíduo já está exercendo equilíbrio, porém vários exercícios podem ser acrescentados para que possa 
aumentar a extensão dos estímulos, dentre eles, pedir para a criança fechar os olhos, realizar exercícios de MMII, retirar os pés do estribo, solicitar que o cavalo pare e ande diversas vezes. Concluíram que, quanto mais precoce inicia-se o tratamento mais ganhos satisfatórios haverão. Em ambos os grupos houve aquisição de marcos motores e equilíbrio, sendo mais evidente no grupo de fisioterapia. Na equoterapia houve reações adaptativas mais rápidas devido ao ambiente, trazendo grandes influências de ajustamento tônico, no entanto menos eficazes. Apesar que, ressaltaram que o tempo de tratamento da equoterapia foi menor que o da fisioterapia.

O equilíbrio é um componente importante do funcionamento das funções motoras, e sabe-se que a hipotonia muscular e o atraso do desenvolvimento motor colaboram para o déficit de equilíbrio e de postura. Durante o andar do cavalo, o movimento tridimensional faz com que o praticante montado se ajuste aos movimentos impostos, assim realizando ajustes posturais de tronco, cabeça e membros, auxiliando nas variáveis cinemáticas do caminhar da pessoa com SD, consequentemente melhorando o equilíbrio. Um estudo eletromiográfico analisou qual material de montaria e posicionamento dos pés seriam mais apropriados para os praticantes com SD na equoterapia. Foram realizadas montarias diferentes, em manta e sela, e posicionamentos dos pés no estribo e fora dele. Obtiveram como resultado que a montaria em manta e os pés fora do estribo geram uma maior atividade muscular de tronco, aumentando de força, e que a equoterapia se difere por não trabalhar com um grupo de músculos isoladamente como na fisioterapia, mas sim abrangendo uma gama maior de agrupamentos musculares, trabalhando o paciente como um todo. Oferecendo assim ganho de força muscular e consequentemente uma melhora de equilíbrio, além de aumento de ADM e melhora de coordenação motora (Espíndula et al., 2014)

Para reforçar os efeitos da equoterapia para pessoas com SD, o estudo de Schelbauer e Pereira (2012)., verificou o equilíbrio, tónus muscular, força muscular e motricidade global. Dado o início da pesquisa, foi feita uma anamnese, além de exame físico, constatando força muscular, reflexos tendinosos, tónus muscular, equilíbrio, marcha e motricidade global, foram feitas 10 provas motoras de cada modalidade, e tais características foram avaliadas pré e pós intervenção de equoterapia. Para o uso lúdico, visando a melhora da motricidade, foram usados: bambolê, bola e prendedores. Constatou-se que os resultados foram positivos em todas as áreas propostas, e que a prática de equoterapia é benéfica para ganhos de equilíbrio, tónus muscular e ganho de motricidade em qualquer faixa etária. Devido a prática oferecer diversos estímulos que são enviados para o SNC, o praticante é guiado a deslocar-se juntamente com os movimentos do equino, assim tendo que criar um equilíbrio e favorecendo a motricidade. 
Esta revisão destaca a importância de se utilizar instrumentos de avaliação precisos para verificar os resultados da terapia realizada. Em um estudo, a foto biometria foi escolhida para a análise de oscilação do corpo, antes e após o tratamento equoterapêutico. Após 16 atendimentos de equoterapia com manta e pés no estribo, em terreno plano de areia, os graus de oscilações diminuíram consideravelmente, tanto em plano frontal quanto sagital. Com olhos abertos verificou-se a diminuição de 19 para 18 graus à medida que, sem visão a melhora foi mais significativa de 21 graus para 14. No plano sagital com graus de 29 para 24 graus com visão e sem visão de 32 graus para 23. Houve também um melhor alinhamento biomecânico diante dos exercícios. Este estudo ressalta que a equoterapia traz benefícios multifatoriais (Meneghetti, Porto, Iwabe \& Poletti, 2009).

\section{CONCLUSÃO}

A equoterapia proporciona benefícios significantes em pacientes com Síndrome de Down como: melhora de equilíbrio corporal, aumento de tónus muscular, entre outras. Assim como propicia um melhor desenvolvimento psicossocial pois, o praticante cria um laço afetivo com o animal, fazendo com que o exercício seja prazeroso, além de ser uma terapia pouco casual proporcionando ao paciente o contato com o animal e o meio ambiente.

Ao longo deste trabalho foram mostradas diferentes atividades na equoterapia, como envolver a criança com brincadeiras lúdicas, alguns dos objetos utilizados e como o tipo de material de montaria pode interferir nos resultados do atendimento: como o uso da manta ou sela, os pés no estribo ou fora dele, bem como a comparação entre equoterapia e fisioterapia convencional, ambas divergem opiniões, assim carecendo de mais pesquisas para comprovação de qual seria a mais apropriada.

Conclui-se que a equoterapia vem sendo aprimorada cada vez mais e que são grandiosos os resultados sobre a melhora do equilíbrio estático e dinâmico dos indivíduos com SD. Porém, ainda há a necessidade de mais pesquisas para ressaltar o que de melhor ela tem a oferecer, não só para a SD, mas também para outras patologias. 


\section{REFERÊNCIAS}

Barreto, F., Gomes G., Silva, I. A. S. \& Gomes, A. L. M (2007). Proposta de um programa multidisciplinar para portador de síndrome de down através de atividades da equoterapia, a partir dos princípios da motricidade humana. Fitness \& Performance Journal, 6(2), 82-88.

Borssatti, F., Anjos, F. B. \& Ribas, D. I. R (2013). Efeitos dos exercícios de força musc na marcha de indivíduos portadores de síndrome de down. Fisioterapia em Movimeto, 26(2), 329335.

Cardozo, J. F. \& Vespasiano, B (2012). Equoterapia para síndrome de down. Revista Científica Eletrônica de Ciências Aplicadas-FAlT, 12(1), 1-3.

Copetti, F., Mote, C. B., Graup, S., Menezes K. M. \& Venturini, E. B (2007). Comportamento angular do andar de crianças com syndrome de down após intervenção com equoterapia. Revista Brasileira de Fisioterapia, 11(6), 503-507.

Espindula, A. P., Ribeiro M. F., Souza, L. A. P. S., Ferreira, A. A. \& Teixeira, V. P. A (2015). Avaliação muscular eletromiográfica em pacientes com síndrome de down submetidos a equoterapia. Revista Neurociência, 23(2), 218-226.

Espíndula, A. P., Assis, I. S. A., Simões, M., Ribeiro, M. F., Ferreira, A. A., Ferraz, P. F., Cunha, I. C., Ferraz, M. L. da F., Souza, L. A. P. S. de, Bevilacqua, E., Jr. \& Teixeira, V. de P. A (2014). Material de montaria para equoterapia em indivíduos com síndrome de down: estudo eletromiográfico. ConScientiae Saúde, 13(3), 349-356.

Gimenez R., Stefanoni F. F. \& Farias P. B (2007). Relação entre a capacidade de sincronização temporal e os padrões fundamentais de movimento rebater e receber em indivíduos com e sem síndrome de down. Revista Brasileira de Ciência e Movimento, 15(3), 95-101.

Granados A. C. \& Agís I. F (2011). Why children with special needs feel better with hippotherapy sessions: A conceptual review. The Jornual of Alternative And Complementary Medicine, 17(3), 191-197. 
Lima J. R. \& Miyagawa M. Y (2007). A influência da equoterapia no tratamento dos distúrbios de equilíbrio em portadores de síndrome de down. Trabalho de Conclusão de Curso, Universidade da Amazônia Unama, Belém.

Liporoni G. F \& Oliveira A. P. R (2005). Equoterapia como tratamento alternativo para pacientes com sequelas neurológicas. Revista Científica Universidade Franca, 5(1/6), 21-29.

Meneghetti C. H. Z., Porto C. H. S., Iwabe C. \& Poletti S (2009). Intervenção da equoterapia no equilíbrio estático de criança com síndrome de down. Revista Neurociência, 17(4), 392396.

Schelbauer C. R. \& Pereira P.A (2012). Efeito da equoterapia como recurso terapêutico associado com a psicomotricidade em pacientes portadores de síndrome de down. Saúde Meio Ambiente, 1(1), 117-130.

Silva J. P. \& Aguiar O. X (2008). Equoterapia em crianças com necessidades especiais. Revista Científica Eletrônica Psicologia, 11(6), 1-8.

Torquato J. Á., Lança A. F., Pereira D., Carvalho F. G. \& Silva R. D (2013). Aquisição da motricidade em crianças portadoras de síndrome de down que realizam fisioterapia ou praticam equoterapia. Fisioterapia em Movimento, 26(3), 515-524. 